\title{
La cultura alimentaria en el desarrollo de diabetes gestacional en usuarias de control prenatal
}

\section{Food culture in the development of gestational diabetes in prenatal care users}

\author{
Katherine Monserrate Villacreses-Merino \\ villacreses@hotmail.com \\ Universidad Estatal del Sur de Manabí, Jipijapa \\ Ecuador \\ https://orcid.org/0000-0001-7292-0875 \\ Jennifer Selena Quimis-Del-Valle \\ ieniffer kimi@hotmail.com \\ Universidad Estatal del Sur de Manabí, Jipijapa \\ Ecuador \\ https://orcid.org/0000-0001-7555-1921 \\ Génesis Juliana Párraga-Moreira \\ geneparragamoreira.6@gmail.com \\ Universidad Estatal del Sur de Manabí, Jipijapa \\ Ecuador \\ https://orcid.org/0000-0002-7035-685X \\ Mayra Alejandra Muñoz-Triviño \\ myaalejandra1809@gmail.com \\ Universidad Estatal del Sur de Manabí, Jipijapa \\ Ecuador \\ https://orcid.org/0000-0002-3691-8409
}

Recibido: 10 de julio de 2021

Aprobado: 15 de octubre de 2021 


\title{
RESUMEN
}

El objetivo general de la presente investigación fue analizar la cultura alimentaria en el desarrollo de diabetes gestacional en usuarias de control prenatal. Se desarrolló mediante un estudio de tipo descriptivo que permitió detallar con fundamentos teóricos, de corte transversal dado que la recolección de datos se efectuó en un tiempo determinado, se utilizó la técnica de encuestas a usuarias que acudieron al servicio de ginecología del establecimiento de salud. Los resultados de la aplicación de la encuesta se recopilaron y procesaron para su posterior tabulación a través del sistema estadístico SPSS 25, la población se conformó por 451 gestantes que acudieron a consulta en el mes de enero 2020, la muestra se escogió aleatoriamente al azar constituida por 85 embarazadas. Se determinó la incidencia de diabetes gestacional en el Establecimiento de Primer Nivel en Salud Jipijapa, con un resultado del 7,24\% en el desarrollo de la patología en gestantes.

Descriptores: Embarazo; salud de la mujer; bienestar de la madre. (Tesauro UNESCO).

\begin{abstract}
The general objective of this research was to analyze food culture in the development of gestational diabetes in prenatal care users. It developed Through a descriptive study that allowed detailing with theoretical foundations, cross-sectional since the data collection was carried out in a certain time, the technique of questioning users who attended the gynecology service of the health establishment was used. The results of the application of the survey were collected and processed for later tabulation through the SPSS 25 statistical system, the population was made up of 451 pregnant women who came for consultation in January 2020 , the sample was randomly chosen at random constituted for 85 pregnant women. The incidence of gestational diabetes was determined in the Jipijapa First Level Health Establishment, with a result of $7.24 \%$ in the development of the pathology in pregnant women.
\end{abstract}

Descriptors: Pregnancy; women's health; mother's welfare. (UNESCO Thesaurus). 


\section{INTRODUCCIÓN}

El embarazo es un proceso complejo que pasa por diversas etapas hasta el desarrollo total y completo de un nuevo ser vivo, durante este periodo los cambios que se producen en la madre son de gran influencia para la salud prenatal, estos ayudan a prevenir en su mayoría la presencia de enfermedades y permiten un adecuado desarrollo y protección en la salud del bebé. Así mismo, Parada (2019) indica lo siguiente:

En los últimos 25 años, el cuidado prenatal y del parto de calidad está en la agenda como una estrategia crucial para reducir la morbilidad y mortalidad materna e infantil. La enfermería, como área de conocimiento, tiene mucho que aportar en esta dirección, así como en el logro de la cobertura universal de los servicios esenciales de salud. (p.6)

Conforme transcurre el embarazo es indispensable suplir los requerimientos de la madre en base a su estado nutricional, enfocado en una alimentación completa, variada, equilibrada tanto en cantidad como en calidad, que brinden los nutrientes, energía y suplementos necesarios para optimizar la salud materna y asegurar el adecuado desarrollo neonatal. Esta etapa está llena de transformaciones físicas, psicológicas y emocionales, con sus correspondientes cuidados, llenos de restricciones/prohibiciones. (Andina Díaz, 2021, p.99)

En este sentido, la nutrición y salud de la gestante ocupa un papel significativo en el bienestar y desarrollo fetal, sin embargo; el entorno, creencias sociales y culturales interviene en la adopción inadecuada de prácticas alimentarias, que inducen a una alteración en la distribución y metabolización de los alimentos que generan consigo un déficit o exceso de peso, desencadenantes iniciales de complicaciones obstétricas. Al respeto, Frías Ordoñez, Pérez Gualdrón y Saavedra Ortega (2016) manifiestan:

La diabetes mellitus gestacional (DMG) ocurre cuando la secreción de insulina no es suficiente para compensar la disminución en la sensibilidad a esta; en su fisiopatología se ven relacionados mecanismos mediados de modo autoinmune, anormalidades genéticas en la función de las células beta $\beta$ pancreáticas 0 deterioro en la resistencia a la insulina. (p.169) 
Las mujeres en estado gestacional, en su mayoría poseen hábitos pocos saludables como el sedentarismo causado por la falta de actividad física, malnutrición por exceso en la ingesta de carbohidratos y grasas saturadas, que aumentan el riesgo de desarrollar sobrepeso u obesidad y comprometen la salud tanto de la madre como del producto, esto conlleva a complicaciones de macrosomía fetal, defectos del tubo neural y el desarrollo de enfermedades metabólicas en el bebé, mientras que en la mujer aumenta el riesgo de hipertensión, hiperglucemias y trastornos del metabolismo de carbohidratos, factores que se relacionan al desarrollo de diabetes gestacional. Por ello, Pando Hernández, et al. (2019) indican:

La DG se ha asociado con una mayor frecuencia de complicaciones maternas y perinatales. Entre las primeras están: una alta incidencia de preclamsia, índices de operación cesárea más elevados y Diabetes mellitus tipo 2 en períodos posteriores al parto; entre las segundas, se señalan: la macrosomía, hipoglucemia, mayor riesgo de obesidad en la infancia y adolescencia. (p.4)

La Organización Mundial de la Salud define a la diabetes gestacional como una hiperglucemia transitoria que se manifiesta o identifica únicamente durante el embarazo y desaparece con el postparto (OMS,2016a). Los elevados niveles de azúcar en la sangre durante el embarazo es una condición de la salud pública presente a nivel global considerada potencialmente de alto riesgo para la gestante y para el desarrollo normal del bebé en el vientre materno, la cual requiere de una valoración, seguimiento y atención multidisciplinaria e integral con enfoque ginecológico y nutricional, que permitan disminuir la morbi-mortalidad materna-neonatal. Por ello es importante, tener en cuenta que consideran los siguiente autores Pereira Despaigne, et al. (2015):

El diagnóstico temprano de la DM favorece el inicio del tratamiento para la prevención de complicaciones. En países en vías de desarrollo, esta afección se diagnostica en menos de $50 \%$ de quienes la padecen y en alrededor de $25 \%$ de las personas en las cuales ha sido recién confirmada, se presenta alguna complicación microvascular. (p.555) 
En este sentido, es recomendable, el asesoramiento sobre hábitos saludables de alimentación, nutrición óptima y qué vitaminas o minerales deben tomar las mujeres durante el embarazo puede contribuir sobremanera a ayudar a que las mujeres y sus niños en desarrollo se mantengan sanos a lo largo del embarazo y después de él», indicó el Dr. Francesco Branca, Director del Departamento de Nutrición para la Salud y el Desarrollo de la OMS. (Organización Mundial de la Salud,2016b).

Los pacientes deben ser capaz practicar el autocontrol mediante la determinación de mediante exámenes periódicos, lo siguiente: a) Glucosuria, b) Cuerpos cetónicos en la orina, c) Glicemia capilar, d) Perfil glicémico y e) También es conveniente la determinación de la hemoglobina glucosilada (HbA1). (Romero Frómeta,2018, p.380)

Por lo tanto, el Ministerio de Salud Pública del Ecuador, en su guía práctica clínica para la alimentación y nutrición en la mujer embarazada, hace énfasis en que las mujeres en su etapa reproductiva deben realizarse obligatoriamente el tamizaje para el diagnóstico de diabetes gestacional entre las 24 y 28 semanas. (Ministerio de Salud Pública del Ecuador, 2014). Es aconsejable para el análisis integral de las futuras madres tomar en consideración lo que plantean Laza Vásquez y Cárdenas, Fernando (2008):

La cultura de un pueblo determina el significado y visión de la vida, la salud, la enfermedad, la muerte y las prácticas de cuidado. La gestación, la maternidad y el nacimiento no están exentos de las construcciones culturales, sus valores y creencias en torno a este proceso, considerado como natural y que se enmarca en la continuidad de la raza humana. (p.3)

De acuerdo a lo esbozado se presenta como objetivo general de la investigación analizar la cultura alimentaria en el desarrollo de diabetes gestacional en usuarias de control prenatal. 


\section{METODOLOGÍA}

El desarrollo de la investigación se realizó mediante un estudio de tipo descriptivo que permite detallar con fundamentos teóricos como la cultura alimentaria de las embarazadas cumple un papel predisponente en el desarrollo de la diabetes gestacional, es de corte transversal dado que la recolección de datos se efectuó en un tiempo determinado, analítico porque nos permitió conocer a profundidad el objeto de estudio, observar las causas, naturaleza y efectos que tienen los hábitos alimenticios en las gestantes y cuantitativo al recopilar datos cuantificables, permitiéndonos en base a esto ejecutar el análisis estadístico.

Para la obtención de datos se redactó una solicitud dirigida a la dirección Distrital 13D03 Jipijapa-Puerto López para la apertura y ejecución del trabajo investigativo en el Establecimiento de Primer Nivel en Salud Jipijapa, la técnica del estudio aplicada fue a través de la utilización de encuestas a usuarias que acudieron al servicio de ginecología por sus controles prenatales, el instrumento aplicado estuvo estructurado por 6 preguntas diseñadas en base al objetivo planteado para la elaboración del proyecto investigativo. Los resultados de la aplicación de la encuesta se recopilaron y procesaron para su posterior tabulación a través del sistema estadístico IBM SPSS 25 de acuerdo a las normas y procedimientos de las ciencias estadísticas se elaboraron tablas, procediendo al análisis e interpretación de los resultados. La población se conformó por 451 gestantes que acudieron a consulta externa en el mes de enero 2020, la muestra se escogió aleatoriamente al azar constituida por 85 embarazadas que acudieron a sus controles prenatales. 


\section{RESULTADOS}

A continuación, se muestra los resultados obtenidos producto de la encuesta aplicada a un grupo de gestantes, acudieron a consulta de control prenatal.

\section{Cuadro 1.}

Edad.

\begin{tabular}{ccccc}
\hline & & Frecuencia & Porcentaje & Porcentaje válido \\
\hline \multirow{4}{*}{ Válido } & 15-20 años & 18 & $\mathbf{2 1 , 2}$ & 21,2 \\
& 21-30 años & 49 & $\mathbf{5 7 , 6}$ & 57,6 \\
& $41-50$ años & 14 & $\mathbf{1 6 , 5}$ & 16,5 \\
& Total & 4 & $\mathbf{4 , 7}$ & 4,7 \\
\hline
\end{tabular}

Elaboración: Los autores.

En lo referente a la edad de las gestantes encuestadas, se puede evidenciar que existe un mayor incide de usuarias jóvenes que acuden a sus controles prenatales entre los 15 y 30 años con un porcentaje acumulado de 78,8\% a diferencia de las usuarias entre los 31-50 años con un menor índice de embarazos en un porcentaje acumulado de $21,2 \%$. La diabetes gestacional se puede presentar a cualquier edad en la fase reproductiva dado que durante este periodo las mujeres producen las mismas hormonas placentarias para permitir el adecuado desarrollo y crecimiento fetal, que interviene directamente entre los mecanismos fisiopatológicos en el desarrollo de dicha condición, sin embargo; se considera 
un mayor grupo de riesgo en la patología aquellas mujeres de edad materna avanzada, considerándose a partir de los 35 años, debido a que la fertilidad durante este periodo de vida decrece exponencialmente, y vulnerabiliza al desencadenamiento de distintas enfermedades prenatales.

\section{Cuadro 2.}

Semanas de gestación.

\begin{tabular}{|c|c|c|c|c|}
\hline & & Frecuencia & Porcentaje & $\begin{array}{c}\text { Porcentaje } \\
\text { válido }\end{array}$ \\
\hline \multirow{4}{*}{ Válido } & $\begin{array}{l}\text { Primer Trimestre } \\
\text { (1-12 semanas) }\end{array}$ & 25 & 29,4 & 29,4 \\
\hline & $\begin{array}{l}\text { Segundo } \\
\text { Trimestre (13-26 } \\
\text { semanas) }\end{array}$ & 36 & 42,4 & 42,4 \\
\hline & $\begin{array}{l}\text { Tercer Trimestre } \\
(27-40 \\
\text { semanas })\end{array}$ & 24 & 28,2 & 28,2 \\
\hline & Total & 85 & 100,0 & 100,0 \\
\hline
\end{tabular}

\section{Elaboración: Los autores.}

Las gestantes encuestadas estuvieron conformadas por diferentes trimestres de embarazo, se consideró la valoración de la edad gestacional como parte del diagnóstico del grupo de riesgo en el desarrollo de diabetes gestacional acorde a lo establecido, ante la pertinente evaluación, se obtuvo como resultado un $42,4 \%$ correspondiente al segundo trimestre y en menor estadística el 28,2\% que pertenece al tercer trimestre de embarazo, considerándose que ambas etapas son puntos de transferencia en el favorecimiento de la presencia de hiperglicemias durante el embarazo, producidas por el aumento de hormonas placentarias 
donde se reduce la sensibilidad de la insulina, es evidente que en la institución se realizan los controles prenatales correspondientes para determinar el estado de salud maternoneonatal, sobre todo en la búsqueda y disminución de los riesgos en la presencia de diabetes gestacional tomando como punto de referencia los trimestres de embarazo. Mientras tanto el $29,4 \%$ pertenece a las gestantes que se encontraban cursando su primer trimestre de gestación en donde se presentan menores índices del desarrollo de la patología, pero se determinan otras acciones encaminadas a cuidar el bienestar y desarrollo sin complicaciones.

\section{Cuadro 3.}

Índice de masa corporal.

\begin{tabular}{|c|c|c|c|c|}
\hline & & Frecuencia & Porcentaje & Porcentaje válido \\
\hline \multirow{6}{*}{ Válido } & $\begin{array}{l}\text { Peso Normal } \\
(18.50-24.99)\end{array}$ & 24 & 28,2 & 28,2 \\
\hline & $\begin{array}{l}\text { Sobrepeso } \\
(25.00-29.99)\end{array}$ & 39 & 45,9 & 45,9 \\
\hline & $\begin{array}{l}\text { Obesidad Grado } \\
\text { I (30.00-34.99) }\end{array}$ & 17 & 20,0 & 20,0 \\
\hline & $\begin{array}{l}\text { Obesidad Grado } \\
\text { II (35.00-40.00) }\end{array}$ & 3 & 3,5 & 3,5 \\
\hline & $\begin{array}{l}\text { Obesidad Grado } \\
\text { III }(>40.00)\end{array}$ & 2 & 2,4 & 2,4 \\
\hline & Total & 85 & 100,0 & 100,0 \\
\hline
\end{tabular}

Elaboración: Los autores. 
Con respecto al índice de masa corporal, se consideró la revisión del carnet prenatal enfocándonos en el peso y talla al inicio del embarazo de cada gestante en estudio, para clasificar el grupo de riesgo en el que se encontraban al desarrollo de la diabetes gestacional, donde se obtuvo resultados alarmantes en la incidencia del sobrepeso con un $45,9 \%$, y mediante la agrupación de las variables de obesidad grado I, II,III con un porcentaje acumulado de $25,9 \%$, en comparación con el $28,2 \%$ que presentaron un peso adecuado, ambos indicadores inquietantes son factores predisponentes en el desarrollo de enfermedades metabólicas entre ellas la diabetes gestacional que al originarse una ganancia de peso conforme transcurre el embarazo produce un acumulo de la adiposidad abdominal e influye en la resistencia de la insulina, de acuerdo con el MSP el peso permisible, durante el segundo y tercer trimestre del embarazo en aquellas gestantes con sobrepeso es de $7-11,5 \mathrm{~kg}$ y en caso de la obesidad mantenerse en un rango de $5-9 \mathrm{~kg}$ (10), para evitar el desencadenamiento de diversas complicaciones que se convierten en un conflicto para la salud pública al incrementar los riesgos obstétricos y neonatales, acorde a los resultados obtenidos por las encuestadas podemos determinar que en la población a nivel local existe una prevalencia de sobrepeso y obesidad en las mujeres de edad reproductiva.

\section{Cuadro 2.}

Conocimiento sobre diabetes gestacional.

\begin{tabular}{ccccc}
\hline & & Frecuencia & Porcentaje & Porcentaje válido \\
\hline \multirow{4}{*}{ Válido } & $\mathrm{Si}$ & 20 & $\mathbf{2 3 , 5}$ & 23,5 \\
& No & 65 & $\mathbf{7 6 , 5}$ & 76,5 \\
& Total & 85 & 100,0 & 100,0 \\
\hline
\end{tabular}

Elaboración: Los autores. 
Se evaluó el nivel de conocimiento de las encuestadas con respecto a una de las variables del tema en investigación, y como resultado se observó que la cifra más elevada con un $76,5 \%$ de las embarazadas que asisten al Establecimiento de Primer Nivel en Salud Jipijapa no tienen conocimiento sobre que es la diabetes gestacional, esta patología a nivel mundial se encuentra entre las principales causas de morbilidad materno-neonatal, de este punto radica la importancia en el conocimiento de la gestante de dicha condición y sus factores desencadenantes durante el embarazo, el $23,5 \%$ de las gestantes que tenían cierto conocimiento de la patología correspondían a estudiantes y profesionales del área de la salud que acudían a sus controles prenatales.

\section{Cuadro 5.}

Antecedentes de diabetes mellitus tipo II.

\begin{tabular}{ccccc}
\hline & & Frecuencia & Porcentaje & Porcentaje válido \\
\hline \multirow{4}{*}{ Válido } & $\mathrm{Si}$ & 61 & $\mathbf{7 1 , 8}$ & 71,8 \\
& No & 24 & $\mathbf{2 8 , 2}$ & 28,2 \\
& Total & 85 & 100,0 & 100,0 \\
\hline
\end{tabular}

Elaboración: Los autores.

Los antecedentes familiares de diabetes mellitus tipo II del grupo encuestado se obtuvo un resultado del $28,2 \%$ sin familiares con dicha patología y una cifra relevante del $71,8 \%$. De acuerdo a estudios recientes por científicos del Instituto de Genética Médica y Molecular de la Paz y del Centro Andaluz de Biología Molecular y Medicina Regenerativa, los antecedentes familiares con diabetes mellitus tipo II que se vincula directamente con parientes de primer grado (padres, hermanos o abuelos) y segundo grado (tíos, sobrinos), demuestran que el factor genético favorece el desencadenamiento de la diabetes gestacional. 


\section{Cuadro 6.}

Realización de prueba de glucosa durante el embarazo.

\begin{tabular}{ccccc}
\hline & & Frecuencia & Porcentaje & Porcentaje válido \\
\hline \multirow{3}{*}{ Válido } & $\mathrm{Si}$ & 53 & $\mathbf{6 2 , 4}$ & 62,4 \\
& No & 32 & $\mathbf{3 7 , 6}$ & 37,6 \\
& Total & 85 & 100,0 & 100,0 \\
\hline
\end{tabular}

Elaboración: Los autores.

El $62,4 \%$ de las gestantes encuestadas manifestaron que si se han practicado pruebas de glicemia durante el embarazo, entre ellas un 3,5\% obtuvieron cifras elevadas al sobrepasar los $120 \mathrm{mg} / \mathrm{dl}$, la práctica del test $\mathrm{O}$ 'Sullivan es una prueba de glucosa realizada en la semana 24-28 con una repetición en la semana 32-34 durante la gestación, sin embargo, en aquellas mujeres con presencia de dos o más factores de riesgo en desarrollar diabetes gestacional, determinado por medio de la anamnesis y examen físico esta prueba se la puede realizar en cualquier momento del embarazo, se logró comprobar que en el establecimiento de atención primaria en salud donde la población en estudio realizan sus controles prenatales se está llevando a cabo los lineamientos correspondientes a la atención prenatal en la búsqueda de la reducción de las enfermedades materno-neonatales, mientras que el 37,6 por ciento de gestantes que aún no se les practica dicha prueba pertenecen al grupo de embarazada que no poseen un riesgo en desarrollar diabetes gestacional y no cumplen aun las semanas establecidas para la realización de dicha prueba. 


\section{CONCLUSIÓN}

El presente estudio permitió conocer que, entre las gestantes que acudieron a su control prenatal durante la investigación un $71,8 \%$ presentaron IMC elevado, este indicador es un factor de riesgo notable al destacar la presencia de obesidad y el sobrepeso ligadas a una inadecuada alimentación, convirtiéndose directamente en un grupo vulnerable al desarrollo de la diabetes gestacional.

Mediante la recolección de los datos estadísticos se determinó la incidencia de diabetes gestacional en el Establecimiento de Primer Nivel en Salud Jipijapa, con un resultado del $7,24 \%$ en el desarrollo de la patología en gestantes que llevan su control prenatal en dicha institución, que corresponde un porcentaje alto en este grupo poblacional, lo cual constituye un problema de Salud Pública prioritario por la exposición de complicaciones en la salud materno-neonatal.

\section{REFERENCIAS CONSULTADAS}

Andina Díaz, E., Martins, F., y Siles González, J. (2021). Creencias y prácticas alimentarias en embarazo y puerperio: aplicación del Modelo de Tradiciones de Salud. Enfermería Global, 20(61), 98-121. Recuperado de: https://dx.doi.org/10.6018/eglobal.413651

Frías Ordoñez, J., Pérez Gualdrón, C., Saavedra Ortega, D. (2016). Diabetes mellitus gestacional: una aproximación a los conceptos actuales sobre estrategias diagnósticas. [Gestational Diabetes Mellitus: a review of current diagnostic strategies concepts]. Rev. Fac. Med. 64(4):769-75. doi: http://dx.doi.org/10.15446/revfacmed.v64n4.54569

Laza Vásquez, C., y Cárdenas, F. (2008). Una mirada al cuidado en la gestación desde la enfermería. Revista Cubana de Enfermería, 24(3-4) Recuperado de: https://n9.cl/1qdg1

Ministerio de Salud Pública del Ecuador.(2014) Alimentación y nutrición de la mujer gestante y la madre en periodo de lactancia. Guía de Práctica Clínica (GPC). Guía de Práctica Clínica (GPC). Quito, Ecuador: : Ministerio de Salud Pública, Subsecretaria Nacional de Gobernanza de la Salud; 2014. Report No.: IBSN-9789942-07-733-2. 


\section{Revista Interdisciplinaria de Humanidades, Educación, Ciencia y Tecnología}

Año VII. Vol. VII. N². Edición Especial II. 2021

Hecho el depósito de ley: pp201602FA4721

ISSN-L: 2542-3029; ISSN: 2610-802X

Universidad Nacional Experimental Francisco de Miranda (UNEFM). Santa Ana de Coro. Venezuela

Katherine Monserrate Villacreses-Merino; Jennifer Selena Quimis-Del-Valle; Génesis Juliana Párraga-Moreira

Mayra Alejandra Muñoz-Triviño

Organización Mundial de la Salud. (2016a) La OMS señala que las embarazadas deben poder tener acceso a una atención adecuada en el momento adecuado. Recuperado de: https://n9.cl/wl1d

Organización Mundial de la Salud. Organización Mundial de la Salud.(2016b). Recomendaciones de la OMS sobre la atencion prenatal. Recuperado de: https://n9.cl/zf26b

Pando Hernández, E., Miló Valdés, C., Llanio González, R., Llanio González, M., y Gonzalez Ungo, E. (2019). Diabetes gestacional compensada con insulina. Universidad Médica Pinareña, 15(2), 194-204. Recuperado de: https://n9.cl/5rabo

Parada CMGL. Women's health during pregnancy, childbirth and puerperium: 25 years of recommendations from international organizations. Rev Bras Enferm. 2019;72(Suppl 3):1-2. doi: http://dx.doi.org/10.1590/0034-7167-2019-72suppl301

Pereira Despaigne, O., Palay Despaigne, M., Rodríguez Cascaret, A., Neyra Barros, R., y Chia Mena, M. (2015). Hemoglobina glucosilada en pacientes con diabetes mellitus. MEDISAN, 19(4), 555-561. Recuperado de: https://n9.cl/6n9ze

Romero Frómeta, R., Palomares Pickering, L., y Delgado Grenms, L. (2018). Manejo integral de la diabetes durante el embarazo. Revista Información Científica, 97(2), 377-386. Recuperado de: https://n9.cl/yf8op 